\title{
Ocorrência de acidentes ocupacionais em uma clínica escola do estado de São Paulo: estudo retrospectivo
}

Occurrence of occupational accidents in a teaching clinic in the state of São Paullo: a retrospective study

Ocurrencia de accidentes laborales en una clínica docente del estado de São Paulo: un estudio retrospectivo

Lara Maria Bueno Esteves

ORCID: https://orcid.org/0000-0002-4032-6502 Araçatuba School of Dentistry, Brazil E-mail:1m.esteves@unesp.br

Juliana Rico Pires

ORCID: https://orcid.org/0000-0002-4559-2578 University Center of the Educational Foundation of Barretos, Brazil E-mail: juricopires@yahoo.com.br

Eliane Marçon Barroso

ORCID: https://orcid.org/0000-0003-3423-9055 University Center of the Educational Foundation of Barretos, Brazi E-mail: embarroso@uol.com.br

Fábio Luiz Scannavino

ORCID: https://orcid.org/0000-0001-9523-4763 University Center of the Educational Foundation of Barretos, Brazil E-mail: flscannavino@gmail.com

Fabiano de Sant'Ana dos Santos ORCID: https://orcid.org/0000-0002-7344-6973 University Center of the Educational Foundation of Barretos, Brazil E-mail: fss@uol.com.br

\begin{abstract}
Resumo
Lesões percutâneas com material biológico são frequentes na clínica odontológica sendo fatores de riscos associadas a doenças transmissíveis. Entretanto, acredita-se que o conhecimento sobre a prevenção e os cuidados que devem ser implementados advenham da formação do profissional. Desta forma, o objetivo deste estudo foi avaliar a ocorrência de acidentes perfurocortantes em uma clínica escola de odontologia e propor medidas para prevenir e controlar a ocorrência de perfurações. Trata-se de um estudo retrospectivo realizado por meio de consulta de fichas de notificações dos acidentes perfurocortantes avaliadas em um período de dez anos no curso de odontologia do Centro Universitário da Fundação Educacional de Barretos. Os dados coletados foram analisados através do software Excel ${ }^{\circledR}$ e expressos por meio de estatística descritiva, em frequências relativas. Assim, foram analisados 2952 alunos, com $3 \%$ de notificação, ênfase no sexo feminino $(72,5 \%)$, no terceiro ano de graduação $(68,8 \%)$ com maior frequência na disciplina de clinica integrada (47,5\%). Apesar da ausência de descrição detalhada, $69 \%$ dos casos de acidentes foram ocasionados por instrumento perfurocortante. A ocorrência de acidentes perfurocortantes é expressiva e modificações nos métodos de notificação e de orientação aos alunos são necessários com intuito de viabilizar a comunicação entre a instituição de ensino, órgãos públicos e graduandos.
\end{abstract}

Palavras-chave: Acidentes de trabalho; Notificação de acidentes de trabalho; Biossegurança; Prestadores de cuidado de saúde.

\section{Abstract}

Percutaneous lesions with biological material are frequent in the dental clinic and are risk factors associated with communicable diseases. However, it is believed that knowledge about prevention and the care that must be implemented comes from professional training. Thus, the aim of this study was to evaluate the occurrence of needlestick injuries in a dental school clinic and to propose measures to prevent and control the occurrence of perforations. This is a retrospective study carried out by consulting notification sheets of needlestick injuries evaluated over a period of ten years in the dentistry course of the University Center of the Fundação Educacional de Barretos. The collected data were analyzed using Excel ${ }^{\circledR}$ software and expressed through descriptive statistics, in relative frequencies. Thus, 2952 students were analyzed, with $3 \%$ of notification, emphasis on females $(72.5 \%)$, in the third year of graduation (68.8\%) with greater frequency in the discipline of integrated clinic $(47.5 \%)$. Despite the 
absence of a detailed description, $69 \%$ of the accident cases were caused by a sharp instrument. The occurrence of needlestick accidents is significant and changes in the methods of notification and guidance to students are necessary in order to facilitate communication between the educational institution, government agencies and undergraduates.

Keywords: Work accidents; Work accident notification; Biosafety; Health care providers.

\section{Resumen}

Las lesiones percutáneas con material biológico son frecuentes en la clínica dental y son factores de riesgo asociados a enfermedades transmisibles. Sin embargo, se cree que los conocimientos sobre prevención y los cuidados que se deben implementar provienen de la formación profesional. Así, el objetivo de este estudio fue evaluar la ocurrencia de lesiones por pinchazo de aguja en una clínica de la escuela de odontología y proponer medidas para prevenir y controlar la ocurrencia de perforaciones. Se trata de un estudio retrospectivo realizado mediante la consulta de fichas de notificación de lesiones por pinchazo de aguja evaluadas durante un período de diez años en el curso de Odontología del Centro Universitario de la Fundação Educacional de Barretos. Los datos recolectados fueron analizados mediante el software Excel ${ }^{\circledR}$ y expresados mediante estadística descriptiva, en frecuencias relativas. Así, se analizaron 2952 estudiantes, con 3\% de notificación, énfasis en mujeres (72,5\%), en el tercer año de egreso $(68,8 \%)$ con mayor frecuencia en la disciplina de clínica integrada $(47,5 \%)$. A pesar de la ausencia de una descripción detallada, el $69 \%$ de los casos de accidentes fueron causados por un instrumento punzante. La ocurrencia de accidentes por pinchazos de agujas es significativa y son necesarios cambios en los métodos de notificación y orientación a los estudiantes para facilitar la comunicación entre la institución educativa, los organismos públicos y los estudiantes.

Palabras clave: Accidentes de trabajo; Notificación de accidentes de trabajo; Bioseguridad; Proveedores de salud.

\section{Introdução}

O trabalho desempenha papel fundamental na vida social e psicológica do ser humano e, cada vez mais, é reconhecido como um benefício por proporcionar o atendimento às necessidades humanas. Entretanto, embora apresente condições favoráveis ao indivíduo, o trabalho torna-se uma via de mão dupla, uma vez que também apresenta riscos eminentes ao trabalhador quando não orientado ou cumprido em seu exímio. Assim, os acidentes ocupacionais são considerados como injúrias que ocasionam lesões corporais ou desordens funcionais e perda permanente ou temporária da capacidade de trabalho. (Nogueira, et.al, 2016)

Diversos afazeres colocam o ser humano de frente com situações de risco, podendo ser de natureza física, química, mecânica, biológica, ergonômica ou psicossocial, afetando, diretamente, a vida deste profissional. Diante deste contexto, trabalhadores da área de saúde são extremamente expostos a situações corriqueiras de exposição ocupacional. Dentre elas, os acidentes ocupacionais de trabalho ganham destaque, uma vez que colocam o trabalhador em contato direto com materiais biológicos altamente contaminantes (Miranda, et.al., 2017). Essas exposições podem acarretar riscos de infecção por meio de injurias percutâneas, além de contato da mucosa com sangue, tecidos ou fluidos corporais potencialmente infecciosos advindos de pacientes. Estudos que observaram os acidentes ocupacionais em um serviço de saúde, reportaram que apesar dos profissionais ocuparem uma posição importante na sociedade por assistirem aos indivíduos e suas comunidades, acabam fazendo parte de um grupo vulnerável, expostos a condições insalubres de trabalho (Dias, et.al., 2012).

Diante da gravidade destes acidentes, a Organização Mundial da Saúde (OMS) aponta que os profissionais de saúde estão submetidos a um risco três vezes maior do que a população em geral. Atualmente, estima-se que a cada ano ocorra uma média de 35,7 milhões de trabalhadores de saúde e graduandos em todo o mundo que apresentam o risco de adquirir doenças causadas por microorganismos na corrente sanguínea advindos de contato percutâneo, ganhando ainda mais importância e destaque (Assunção, et.al., 2012; Vieira, et.al., 2013).

O acidente de trabalho, por exposição a material biológico, constitui uma preocupação constante das universidades, devido a quantidade de procedimentos realizados e a inexperiência dos acadêmicos frente ao manuseio de instrumentais perfurocortantes, entretanto, sabe-se que a base do conhecimento e da aplicabilidade da prática de redução de tais acidentes é ensinado aos profissionais durante o seu processo de graduação (Assunção, et.al., 2012). Principalmente na odontologia, os 
acidentes são frequentes em decorrência do trabalho continuo com instrumentos pontiagudos e/ou cortantes, um campo de visão restrito e sujeito a movimentação inesperada do paciente (Barbosa, et.al., 1999; Matsumoto, et.al., 2019)

Desta forma, trabalhos apontam que mais de 60 tipos de patógenos podem ser transmitidos por meio de exposições acidentais, como o vírus da hepatite B (HBV), o vírus da hepatite C e o vírus da imunodeficiência humana (HIV), representando os agentes infecciosos de maior importância devido à gravidade de suas sequelas (Martins, et.al., 2010; Tarantola et.al., 2006).

Entretanto, sabe-se que o risco pode ser diminuído quando implementadas barreiras de proteção individuais e coletivas, porém, embora apresentem efetividade, não são práticas rotineiras empregadas e cobradas na área da saúde (MUROFUSE, et.al., 2008). Sendo assim, o Ministério da Saúde preconiza, como medida imediata após exposição ocupacional, a lavagem exaustiva do local injuriado. Paciente e profissional devem ser submetidos a testes sorológicos para investigar possível infecção e, caso positivo, a quimiprofilaxia deve ser iniciada nas primeiras 24 horas (Oreste, et.al., 2009).

Embora ainda apresentem um número expressivo de contaminação por acidentes ocupacionais, a base desta rotina advém do conhecimento passado e cobrado durante o processo de aprendizagem na graduação. Sabe-se que, como carga curricular, a disciplina de Biossegurança desempenha papel fundamental na construção e prevenção de acidentes perfurocortantes. Esta, visa promover ao futuro profissional o conhecimento dos riscos presentes no ambiente de trabalho, bem como, nos procedimentos que são desenvolvidos, promovendo a correta proteção da saúde do profissional, auxiliar, paciente e demais profissionais que apresentem o risco de contaminação (Leal, 2015; Paiva, et.al., 2017).

O conhecimento do ambiente laboratorial e das atividades desenvolvidas estão intimamente ligados na ocorrência de acidentes, sejam de etiologia biológica, perfurocortante ou química. Entretanto, são mínimas as quantidades de pesquisas que avaliam conjuntamente as características principais para quantificar e discutir o problema e propor medidas que verifiquem como controlar os acidentes biológicos (Lima, et.al., 2017). Além disso, estima-se que os acidentes sejam subnotificados devido à falta de consciência, conhecimento, sentimento de culpa por apresentar uma situação com risco eminente, falta de organização e cuidado adequado, negação da relevância do acidente e até mesmo medo de perder sua função no ambiente trabalhista, apresentando grande risco diante da saúde (Barbosa, et.al., 2017).

Diante deste cenário, o fornecimento de informações de alta qualidade sobre possíveis problemas ocupacionais é essencial para que sejam reconhecidos a gravidades deste problema e as informações de conscientização com o intuito de reduzir, assim, a ocorrência de acidentes ocupacionais. Sendo assim, este trabalho tem por objetivo avaliar a ocorrência de acidentes perfurocortantes em uma clínica escola de odontologia e propor medidas para prevenir e controlar a ocorrência dos mesmos.

\section{Metodologia}

Após aprovação do Comitê de Ética em Pesquisa do Centro Universitário da Fundação Educacional de Barretos ( $n^{\circ}$ 123358/2016), iniciou-se um estudo retrospectivo quantitativo (Umbelino, et. al., 2020) realizado por meio de consulta de fichas de notificações dos acidentes perfurocortantes ocorridos nos últimos dez anos relatados no Curso de Odontologia. Foram consideradas as fichas cujos pacientes assinaram um termo de consentimento livre e esclarecido.

O recrutamento das fichas incluídas na amostra foi realizado por busca direta no arquivo da instituição de acordo com os seguintes critérios: graduandos e profissionais que exerciam função na Instituição e fichas que apresentassem o termo de conscentimento livre e esclarecido assinado.

Através do banco de dados da Instituição, as fichas foram recolhidas, analisadas e realizado a tabulação dos dados com o intuito de avaliar os critérios presentes abaixo: 
- Quantidade de alunos totalizados;

- Quantidade de alunos que sofreram perfurações;

- Profissionais que sofreram perfurações;

- Influência do gênero;

- Ano de graduação;

- Disciplina em que o acidente ocorreu;

- Procedimento que o aluno estava realizando;

- Tipo de material utilizado.

Todas as informações adicionais presente na ficha da Instituição foram levadas em consideração. Fichas que apresentavam dados incompletos também foram recrutadas e tabuladas de acordo com as informações fornecidas.

Os dados coletados foram analisados com o auxílio do Excel $^{\circledR}$ e expressos por meio de estatística descritiva, em frequências relativas sendo submetidos à análise de variância pelo Teste Exato de Fisher $(p \leq 0,05)$.

\section{Resultados}

Em 10 anos, foram analisados 2952 alunos, sendo 995 do gênero masculino e 1957 do gênero feminino que estudaram no Centro Universitário da Fundação Educacional de Barretos, no curso de Odontologia, resultando em 80 notificações de acidentes perfurocortantes, aproximadamente, $3 \%$ do total.

A Tabela 1 apresenta a frequência de acidentes dando maior ênfase ao sexo feminino com 72,5\%, e no terceiro ano de graduação com 68,8 \%. A disciplina de maior destaque foi a Clínica Integrada com 47,5 \%. Os procedimentos ficaram em $60 \%$ com ausência de descrição. Sessenta e noves por cento dos casos analisados apresentaram material perfurocortante como causa, porém não o identificaram.

Tabela 1 - Frequência de acidentes na clínica escola nos anos em estudo.

\begin{tabular}{|c|c|c|}
\hline Variável & Frequência $(n=80)$ & Porcentagem (\%) \\
\hline \multicolumn{3}{|c|}{ Gênero } \\
\hline Feminino & 58 & 72,5 \\
\hline Masculino & 22 & 27,5 \\
\hline \multicolumn{3}{|c|}{ Ano de Graduação } \\
\hline Segundo Ano & 12 & 15,0 \\
\hline Terceiro Ano & 55 & 68,8 \\
\hline Quarto Ano & 8 & 10,0 \\
\hline Não se Aplica & 2 & 2,5 \\
\hline Ausente & 3 & 3,8 \\
\hline \multicolumn{3}{|c|}{ Nome da Disciplina } \\
\hline Ausente & 11 & 13,8 \\
\hline Cirurgia & 7 & 8,8 \\
\hline Diagnóstico & 1 & 1,3 \\
\hline Integrada & 38 & 47,5 \\
\hline Dentística & 11 & 13,8 \\
\hline Endodontia & 4 & 5,0 \\
\hline Periodontia & 8 & 10,0 \\
\hline
\end{tabular}




\begin{tabular}{ccc}
\hline & Procedimento & \\
Ausente & 48 & 60,0 \\
Anestesia & 17 & 21,3 \\
Cirurgia & 3 & 3,8 \\
Dentística & 1 & 1,3 \\
Endodontia & 1 & 1,3 \\
Lavagem de & 1 & 1,3 \\
instrumental & 1 & 1,3 \\
Profilaxia & 8 & 10,0 \\
Raspagem & Tipo de Material & \\
\hline Ausente & 2 & 2,5 \\
Perfuração com & 23 & 28,7 \\
Agulha & 55 & 68,8 \\
Perfurocortante & 5 & \\
\hline
\end{tabular}

* Asterisco simboliza diferença estatisticamente significante entre as variáveis estudadas, para $p \leq 0,05$ (Teste Exato de Fisher). Fonte: Autores.

Por meio do teste exato de Fisher para análise dos dados obtidos, não se observou significância, uma vez que, dentre as 80 fichas utilizadas para análise apenas uma estava preenchida corretamente.

\section{Discussão}

Acidentes ocupacionais são definidos como danos ocorridos devido ao desenvolvimento das atividades em determinado local de trabalho, causando alteração funcional ou lesão corporal (Barbosa, et.al., 2017). No Brasil, apesar de poucas pesquisas relacionadas à categoria de cirurgião-dentista, estudos apontaram que são os principais profissionais de saúde que se acidentaram, justificando-se pelo fato de estarem mais expostos aos riscos de acidentes ocupacionais (Paiva, et.al., 2017; Miotto, et.al., 2012). O presente estudo teve por finalidade analisar as 80 fichas de acidentes perfurocortante ocorridos no curso de odontologia do Centro Universitário da Fundação Educacional de Barretos. Apresenta, também, a função de relatar e intensificar a importância de uma correta notificação e da utilização de Equipamentos de Proteção Individual (EPIs) frente à realização de procedimentos com riscos a exposição, seja sangue ou fluídos corpóreos (Theodoro, et.al., 2009)

Este assunto requer tamanha importância sendo destacado nas contribuições ao aprimoramento das Diretrizes Curriculares Nacionais (DCN) em seu capitulo III do curso de Graduação em Odontologia. O artigo $13^{\circ}$, informa que a formação do cirurgião-dentista se objetiva em dotar o profissional dos conhecimentos requeridos para o exercício de competências específicas, através da promoção do autocuidado para a prevenção de acidentes de trabalho e doenças ocupacionais (Paiva, etl.al., 2017).

Assim, as medidas de precaução universais foram instituídas para minimizar o risco de acidentes e transmissão ocupacional de materiais biológicos. Visam reduzir a exposição, devendo ser utilizadas na manipulação de artigos contaminados com fluidos orgânicos e na assistência a pacientes, independentemente do diagnóstico definido, conforme preconizado pelo Centro de Prevenção e Controle de Doenças dos Estados Unidos (Marziale e Valim, 2011). Este conjunto de medidas é oficialmente reconhecido e recomendado também pelo Ministério da Saúde brasileiro e compreende o âmbito de imunização, lavagem das mãos, uso de Equipamentos de Proteção Individual (EPI), Equipamentos de Proteção Coletiva (EPC), 
descarte correto de perfurocortantes e cuidados com os ambientes e processamentos de artigos contaminados (Ministério da saúde, Brasil, 2018).

No Brasil, existem poucas informações em relação a frequência e a natureza dos acidentes ocupacionais odontológicos. Acredita-se que, uma das razões, é a falta de notificação de casos entre estudantes e profissionais de saúde, que por causa do inconveniente e medo do que o relatório pode trazer, ou por falta de conhecimento sobre a conduta correta e gravidade do acidente, acabam não dando a devida importância. Estima-se que, atualmente, mais de 50\% dos acidentes ocorridos não sejam notificados (Pinelli e Mouta, 2014), uma vez que, em muitos casos, o acidente não ocasiona sintomatologia no momento ou nos dias subsequentes, camuflando, assim, a caracterização da transmissão (Soares, et.al., 2019). Ainda, se faz importante a notificação, para que estimativas de ocorrência de acidentes biológicos sejam divulgadas assim como a letalidade das infecções (Magalhães, et.al., 2015)

Ainda na atualidade, com o cenário de transmissibilidade e a necessidade de autocuidado, é essencial buscar conhecimentos sobre as doenças que os profissionais estão expostos, suas rotas de infecção, modos de transmissão e, principalmente, os padrões de biossegurança, como a correta esterilização e desinfecção dos materiais utilizados, adequada eliminação de resíduos e o respeito pelo meio ambiente, gerando, assim, proteção individual e coletiva. A utilização de EPI é fundamental para uma prática segura, entretanto, só se efetivará quando utilizados corretamente. Biossegurança não se resume somente a normas de prevenção, sua grandiosidade requer dos indivíduos uma formação educacional adequada para uma correta execução de seus objetivos (Lages, et.al., 2015).

Em dois estudos realizados no Irã, $90 \%$ dos graduandos de odontologia afirmaram não relatar seus acidentes. Devido a omissão dos acidentes, as medidas profiláticas pós-exposição não foi possível em muitos casos. Diante dos dados obtidos, os mesmos sinalizaram que é necessário a adoção de estratégias para melhorar o registro de informações. É evidente que para o sucesso da prevenção, requer-se uma orientação constante, de maneira que o conhecimento seja passado de forma prática e consiga ser aplicado no cotidiano, com um ganho significativo na saúde dos profissionais e graduandos envolvidos. As rotinas de biossegurança devem ser estimuladas pelos docentes de odontologia e equipes especializadas, além da parte gestora das instituições de ensino, uma vez que são núcleos formadores de práticas profissionais seguras fundamentadas na vida do acadêmico (Vasconcelos Junior, et. al., 2020; Nascimento, et.al., 2012).

Apesar de todas as informações fornecidas no campus da instituição em questão e de uma ficha de notificação vigente, em sua totalidade houve o uso inadequado da mesma. O levantamento proposto possibilitou a visualização de aspectos que até então não tinham sido identificados, tornando pública a situação dos acidentes com risco biológico. Diante disso, preconiza-se que todas as Instituições de Ensino que possuem Clinica Escola ativa implementem uma ficha de notificação (Anexo I) e criem um Comitê de controle de infecção odontológica responsável por fiscalizar o preenchimento e a aplicação das normas de prevenção. Vale ressaltar que mais do que padronizar, é necessário comprometimento com a formação pessoal para que os espaços e as práticas cotidianas atendam aos requisitos necessários para diminuir os riscos de acidentes (Ribeiro, et. al., 2020; Reis, et.al., 2019).

A odontologia, por sua vez, não se isenta do dever de contribuir para que mudanças ocorram. A partir desta questão, é de suma importância que seja investigado o nível de conscientização dos graduandos acerca da biossegurança e dos riscos durante o atendimento clínico. Vale também atentar sobre a importância da vacinação do graduando, colocando-o em risco de contrair doenças, caso não esteja adequadamente imunizado. No entanto, para que a prática de biossegurança seja possível e eficaz, é necessário a conscientização de todos os envolvidos, informações, sensibilizações, treinamento, fiscalização e disponibilização dos recursos e materiais adequados de modo que salvaguarda a salubridade profissional (Armond, et.al., 2016). 


\section{Conclusão}

A ocorrência de acidentes perfurocortantes é presente em grande escala e sua correta notificação é necessária, uma vez que a presença de subnotificações foi encontrada em grande número. Dessa forma, sugere-se a implementação de estratégias de ensino que visem a correta apresentação da importância do conhecimento e das condutas a serem seguidas, além de uma nova maneira de arquivamento dos dados dos envolvidos em acidentes ocupacionais e histórico dos mesmos. Estas novas características viabilizariam a comunicação entre instituição de ensino e órgãos públicos competentes e designados para acompanhamento e levantamento dos riscos ocupacionais, salvaguardando a integridade física e legal dos alunos e da instituição de ensino. Ainda, sugere-se estudos futuros que avaliem se novas estratégias apresentam eficácia no controle de subnotificações e cuidado com a saúde dos envolvidos.

\section{Referências}

Armond, A. C. V., Gonçalves, P. F., Flecha, O. D., Oliveira, D. W. D., Sampaio, F. C. \& Falcs, S. M. (2016). Biosafety knowledge for the main risk activities involving public servers, students and cleaning staff of the dentistry course of FVJM/Diamantina. RBOL. 3 (2): $32-52$.

Assunção, A. A., Araújo, T. M., Ribeiro, R. B. N. \& Oliveira, S. V. S. (2012). Hepatitis B vaccination and occupation exposure in the healthcare sector in Belo Horizonte, Southeastern Brazil. Rev Saúde Pública. 46 (4):665-73.

Barbosa, A. S. A. A., Diogo, G. A., Salotti, S. R. A. \& Silva Smur (2017). Subnotificação de acidente ocupacional com materiais biológicos entre profissionais de Enfermagem em um hospital público. Rev Bras Med Trab. 15(1): 12-7.

Barbosa M. V. J., Souza A. M., Carvalho L. P. F., Hernandez R. V. T. \& Megda S (1999). Incidência de acidentes com materiais perfurocortantes e fluidos corpóreos no hospital universitário "Alzira Velano". R. Un. Alfenas. 5: 221-225.

Brasil. Ministério da Saúde (2018). Secretaria de Vigilância em Saúde. Protocolo Clínico e Diretrizes Terapêuticas para Profilaxia Pós-Exposição (PEP) de Risco à Infecção pelo HIV, IST e Hepatites Virais. Brasília: Ministério da Saúde.

Dias M. A. C., Machado A. A. \& Santos B. M. O. (2012). Occupational injury involving exposure to biological fluids: a portrait of reality. Medicina. 45 (1): $12-22$.

Lages S. M. R., Santos A. F., Silva Junior F. F. \& Costa J. G. (2015). Formación en odontolo gía: El papel de las instituciones de enseñanza en la prevención de accidentescon exposición a material biológico. Ciencia \& Trabajo.17 (54).

Leal C. A. G. (2015). Biosafety and waste management in health services: the importance in the dentistry professionals' formation in perspective of human and environmental health. Rev. ABENO. 15 (2).

Lima G. M. N., Kawanami G. H., Romeiro F. G. (2017). Perfil das exposições ocupacionais a material biológico entre profissionais de saúde do Hospital de Base de Bauru: medidas preventivas e pós-exposição. Rev Bras Med Trab. 15 (3): 194-9.

Magalhães V. C. S., Oliveira D. L. \& Prado F. O. (2015). Knowledge, risk perception and attitudes of Dentistry students with regard to HIV/AIDS. Rev Gaúch Odontol. 63 (3): 291-300.

Martins A. M. E. B. L.; Pereira R. D. \& Ferreira R. C. (2010). Compliance with occupational postexposure protocol for injuries among dental surgeon. Rev Saúde Pública. 44 (3): 529-40.

Marziale M. H. P. \& Valim M (2011). Avaliação da exposição ocupacional a material biológico em serviços de saúde. Texto \& Contexto Enfermagem. 20: $138-146$.

Matsumoto H, Sunakawa M, Suda H \& Izumi Y (2019). Analysis of factors related to needle-stick and sharps injuries at a dental specialty university hospital and possible prevention methods. J Oral Sci. 61: 164-170.

Miotto M. H. M. B.\& Rocha R. M. (2012). Acidente ocupacional por material perfurocortante entre acadêmicos de odontologia. Rev Bras Promoç Saúde. 25 (1): $97-102$

Miranda F. M. D., Cruz E. D. A., Félix J. C. V., Kalinke L. P., Mantovani M. F. \& Sarquis L. M. M. (2017). Profile of Brazilian workers victims of occupational accidents with biological fluids. Rev Bras Enferm. 70 (5): 1061-8.

Murofuse N. T., Alves D. C. I., Fávero G. C.\& Brotto A. O. (2008). Comportamento dos acadêmicos, docentes e técnicos administrativos da clínica odontológica da Unioeste: riscos ocupacionais e atividades desenvolvidas. Acta Sci. Health Sci. 30 (1): 81-87.

Nascimento L. S., Assunção L. R. S., Júnior N. G. S., Pedreira E. M. Silva RLC (2012). Acidentes com Pérfuro-cortantes na Faculdade de Odontologia da UFPA: Visualização de um Cenário. Rev Odontol Bras Central. 21 (56).

Nogueira A. S., Carvalho B. K. G., Medeiros A. R., Carneiro S. E. R. \& Souza G. C. A (2016). Prevalência e notificação de acidentes de trabalho com exposição a material biológico na odontologia. Rev Ciênc Plural. 2 (1): 102-1. 
Research, Society and Development, v. 11, n. 1, e5011124398, 2022

(CC BY 4.0) | ISSN 2525-3409 | DOI: http://dx.doi.org/10.33448/rsd-v11i1.24398

Orestes-Cardoso S. M., Farias A. B. L., Pereira M. R. M. G., Orestes-Cardoso A. J.\& Cunha Junior I. F. (2009). Needlestick and sharp injuries - prevalence and prophylatic measures among dentistry students. Rev. bras. Saúde ocup. 34 (119): 06-14.

Paiva S. N., Zaroni W. C. S., Leite M. F., Bianchi P. R. \& Pereira T. C. R. (2017). Acidentes ocupacionais com material biológico em Odontologia: uma responsabilidade no ensino. Revista da ABENO. 17 (3): 76-88.

Pinelli C.\& Mouta L. F. G. L. (2014). Occupational exposure to contaminated biological material: perceptions and feelings experienced among dental students. Rev Odontol UNESP. 43 (4): 273-279.

Reis L. A., La-Rotta E. I., Diniz P. B., Aoki F. H. \& Jacksjorge J. (2019). Occupational Exposure to Potentially Infectious Biological Material Among Physicians, Dentists, and Nurses at a University Safety and Health at Work. 10 (4): 445-451.

Ribeiro, W. A., Souza, C. E. A. de L., Conceição, V. L. D. da, \& Evangelista, D. da S. (2020). Work nurse in the prevention of occupational biological risks: a literature review in the hospital area. Research, Society and Development, 9(7),

Soares R. Z., Schoen A. S., Benelli K. R. C., Araújo M. S. \& Neves M. (2019). Analysis of reported work accidents involving healthcare workers and exposure to biological materials. Rev Bras Med Trab. 17 (2): 201-208.

Tarantola A., Abiteboul D. \& Rachline A (2006). Infection risks following accidental exposure to blood or body fluids in health care workers: a review of pathogens transmitted in published cases. Am J Infect Contr. 34: 367-375.

Theodoro E. D., Miotto M. H. M. B., Barcellos L. A. \& Grillo C. H. B. (2009). Accidents at work ans Immunization Schedule in Dental Surgeons. Revista Brasileira de Pesquisa em Saúde. 11(1): 27-32.

Umbelino, K. S. M., Silva, N. S. da Silva, M. C., Campos F. N. L. \& Lolli L. F. (2020) Análise da evolução do preenchimento de prontuários clínicos institucionais com a implantação de uma gestão de risco. Revista da Abeno. 20 (1): 80-90

Vasconcelos Júnior, F. C. F., Barbosa, G. S. L., Mouta, A. A. N., Souza, A. T. da S., Rego, C. de S., Hipolito, L. C., Silva, A. C. B. da, \& Beltrão, R. P. L. (2020). Professional exposure and the use of Personal Protective Equipment: integrative review. Research, Society and Development, 9(8)

Vieira C. D., Santos J. I., Cabral M. M. \& Silva J. F. (2013). Biological exposure-related injuries in dental health-care workers. RGO - Rev Gaúcha Odontol. 61 (4): 581-586. 